ethics of the 'balance' between north and south. The growth of courses in 'developing country psychiatry' at the Institute of Psychiatry in London, Manchester and at Keele is, in my view, an entirely appropriate, if belated, response to the particular training needs of doctors, whether UK or foreign doctors, who plan to work in a developing country. One could speculate about the many reasons for the popularity of such courses at the present time. However, the problems of their assessment and my recent sojourn in East
Africa was a timely reminder that the balance of advantage between north and south is now more evenly weighted than any simplistic understanding of British colonial history, or of the more affluent north, would suggest.

It is, indeed, hoped that there could be a joint meeting between the Royal College of Psychiatrists and the Kenyan Psychiatric Association, which I have little doubt would be instructive to participants from whichever continent they come.

\title{
Community-oriented psychiatric care
}

\author{
Some ideas on training*
}

JosEPH ConNolly, Consultant Psychiatrist and IsAac Marks, Professor of Experimental
Psychopathology, The Maudsley Hospital and Institute of Psychiatry, London SE5

The College is debating how to train psychiatrists for community care (CC) that is spreading - ahead of hard evidence of its value for certain problems in the UK. Much future psychiatry will be practised in the community outside hospital within multidisciplinary teams not always led by a psychiatrist, and wherein the lead-rôle changes frequently within a single meeting depending on whose expertise and readiness to accept responsibility emerge.

The following is a list of preliminary ideas on how to equip trainees to work as community psychiatrists, making cogent and valued contributions to team-working. Academic and practical training is needed in most forms of mental health care delivery in diverse settings. Many of the tentative ideas for training to work with adults in CC also apply to the very young and the elderly, to other forms of care and to other care providers. Hands-on experience is required, as key worker for part of the team's caseload, in (6) to (13) following.

* Based on invited talk to and feedback from Open Forum of Royal College of Psychiatrists Education and Collegiate Trainees' Committees, March 1988.
Knowledge is needed of:

(1) the aim of CC to manage mental health problems in the least restrictive settings possible without losing the security, time continuity and depth obtainable in hospital care;

(2) the epidemiology of psychiatric disorder, relating it to the social geography of the community served (rural-urban differences, the homeless and migrants, etc);

(3) the biopsychosocial aspects of causes, presentations, management and outcome of the problems most likely to be seen, distinguishing observation from inference;

(4) the performance - criteria in cost-effective care delivery for the population served;

(5) how to make the most of the team's human resources, knowing each type of carer's core role and shared roles;

(6) how to (a) define the patient's main problems and personal, family, social and physical health context; (b) help each problem using team resources sensibly, taking account of patients (i) coming into the system, (ii) already in it, and (iii) likely to present in future; (c) rapidly summarise the salient features of (a) and (b) for clinical management and communication with other carers; 
(7) the physical and psychological interventions of use across different settings, including (a) crisis resolution, (b) prevention and management of violence, (c) assertive followup and outreach, (d) activities useful in day care, (e) family work and carer-support;

(8) the rapid evaluation of patients' progress: (a) what improves, gets worse, remains unchanged? - among the main problems of selfcare, work, family, leisure and social adjustment, housing, finance, physical health; (b) the time spent to obtain that progress - in direct and indirect care from each carer;

(9) how to keep coordinated care records for individual patients (e.g. use of shared care card), tracking of the whole caseload;

(10) how to access and liaise with further resources outside the team: CPNs and GPs (sessions in primary care with patients and with carers), day care, drop-in centres, social services, educational services, voluntary bodies, housing organisations, Samaritans, police, clergy, $\mathrm{CHC}$, DGH;

(11) best sites in the neighbourhood to give different levels (intensities) of short- and long-term care according to the type of problem (intensive, secure and respite beds; other services providing day care, drop-in centres, outpatient-sessions, home and worksite visiting);

(12) education of patients, family and others about the nature of patients' problems and their care, prevention and early signs of relapse, and return to care if lapsed;

(13) how to work with: (a) Mental Health Act provisions regarding detention, leave, aftercare, noncompliance and possible future guardianship arrangements; (b) Disabled Persons Act 1986 regarding patients' welfare rights, green card entitlement to employment and housing, grants for settling into new housing, security.

Registrars should learn (1) to (13), working, after they have acquired some knowledge of phenomenology and treatment (between 6-18 months into their training), for at least nine months in CC-less time is too short to get a longitudinal view of patients' progress and problems. They should work with an accredited community service in a multidisciplinary team with a more senior psychiatrist acting as mentor, and, under his supervision, as a key worker for some patients but involved with the entire care network. Knowledge and competence in (1) to (13) should be tested as part of the Part II MRCPsych clinical, essay and oral examinations; this would include two 500-word case summaries (of a success and a failure of community care) with brief outcome measures and analysis of the total time spent and methods used by the team to attain that outcome.

Senior Registrars need to learn how to:

(1) train registrars and others in the above

(2) do domiciliary and primary care consultations

(3) manage a caseload

(4) manage a budget

(5) work in care teams and with patient groups

(6) plan and develop community services in the light of their epidemiological base, and how to bid for funds for health and social care

(7) evaluate any changes made in a care system

(8) do routine quality assurance

(9) learn about NHS and Social Service management

(10) outreach to community organisations about possible prevention.

(1) to (10) should be learned while working for a year under an accredited consultant within community teams in both primary and day care, and liaising with DGH, Accident and Emergency, and with residential and voluntary services.

Problems to be addressed include:

(1) smaller catchment areas may have too few posts available to offer a sufficiently varied training

(2) trainees working in community posts may have less chance to interact with peers and so may feel isolated

(3) future clinical scientists may have less time to develop research skills

(4) is the current sharp division between registrar and senior registrar training appropriate? 